\title{
APPROXIMATION OF CONVEX BODIES BY TRIANGLES
}

\author{
MAREK LASSAK
}

(Communicated by William J. Davis)

\begin{abstract}
We show that for every plane convex body $C$ there exist a triangle $T_{1}$ and its image $T_{2}$ under a homothety with ratio $\frac{5}{2}$ such that $T_{1} \subset C \subset T_{2}$. We prove the conjecture of Grünbaum that if $C$ is centrally symmetric, then $T_{1}, T_{2}$ can be chosen so that their centroids coincide with the center of $C$.
\end{abstract}

Let $C$ be a convex body in the Euclidean plane $E^{2}$. By the centroid of $C$ we understand the center of mass of $C$. Of course, if $C$ is centrally symmetric, the centroid is the center of symmetry of $C$. Denote by $\lambda C$ the homothetic copy of $C$ with homothety center at the centroid of $C$ and homothety ratio $\lambda$. By $w(C, l)$ we denote the width of $C$ in the direction $l$.

By an affine-regular hexagon we mean an affine image of the regular hexagon. Besicovitch [2] proved that an affine-regular hexagon can be inscribed in every plane convex body.

Lemma. Let $M \subset E^{2}$ be a centrally symmetric convex body. We can inscribe in $M$ an affine-regular hexagon $H$ whose center is the center of symmetry of $M$ such that $M \subset \frac{3}{2} H$.

Proof. Lemma 1 of [5] says that for every direction $l$ there is exactly one affine-regular hexagon $H(l)=a b c d e f$ (with vertices $a, b, c, d, e, f$ in the positive order) inscribed in $M$ such that the vector $\overrightarrow{a c}$ has direction $l$, and that the vertices of $H(l)$ vary continuously along the boundary of $M$ as $l$ rotates. We define the direction $l^{*}$ associated with $l$ as the direction of the vector $\overrightarrow{a p}$, where $p$ is the perpendicular projection of $a$ on the straight line through $c$ and $d$. It is clear that $l^{*}$ is defined for every direction $l$ and that $l^{*}$ depends continuously on $l$.

Since $M$ is centrally symmetric, the center of symmetry of $H(l)$ is always in the center $o$ of symmetry of $M$ (see the proof of Lemma 1 of [5]).

Denote by $m$ the direction of $\overrightarrow{b d}$. Of course, $H(l)=H(m)$. There is a supporting line of $M$ through $d$. The intersection of this line with the line containing the segment $b c$ is denoted by $v$, and with the line containing $e f$ by $z$ (see Figure 1). Let $Q$ denote the convex cone with vertex $d$ and bounding lines through $c$ and $e$. Since $H(l)$ is inscribed in $M$, the set $M \backslash Q$ is contained in the union of triangles $c d v$ and $d e z$. Moreover, an argument

Received by the editors February 22, 1990 and, in revised form, June 18, 1990.

1980 Mathematics Subject Classification (1985 Revision). Primary 52A10. 


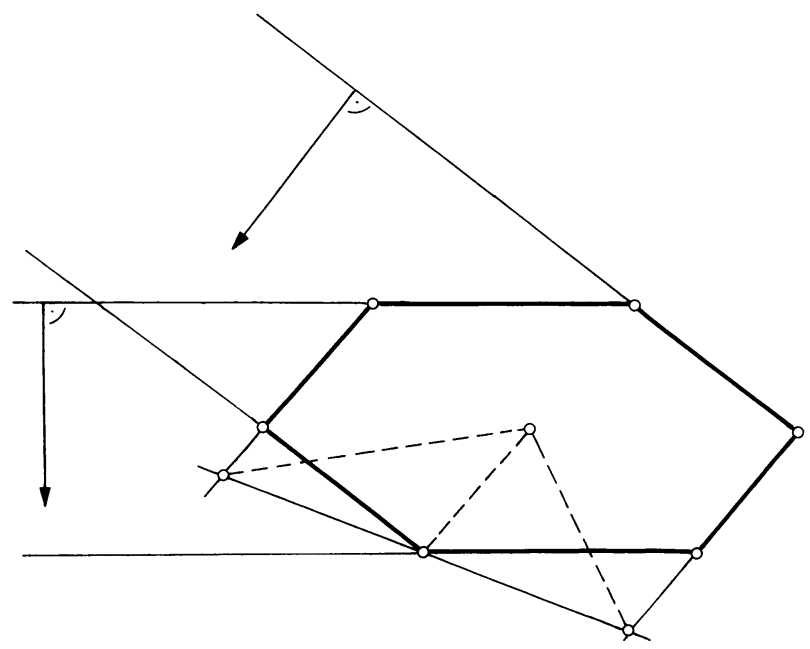

FIGURE 1

from plane geometry establishes that

$$
\text { if } w\left(o v, l^{*}\right) \geq \frac{3}{2} w\left(o d, l^{*}\right), \text { then } w\left(o z, m^{*}\right) \leq \frac{3}{2} w\left(o d, m^{*}\right) \text {. }
$$

Both the facts and the central symmetry of the construction imply

$$
\text { if } w\left(M, l^{*}\right) \geq \frac{3}{2} w\left(H(l), l^{*}\right) \text {, then } w\left(M, m^{*}\right) \leq \frac{3}{2} w\left(H(l), m^{*}\right) \text {. }
$$

Consider the case when there exists a direction $l_{0}$ such that

$$
w\left(M, l_{0}^{*}\right)>\frac{3}{2} w\left(H\left(l_{0}\right), l_{0}^{*}\right) ;
$$

in the opposite case Lemma is trivially true.

Denote by $m_{0}$ the direction of $\overrightarrow{b_{0} d_{0}}$, where $H\left(l_{0}\right)=a_{0} b_{0} c_{0} d_{0} e_{0} f_{0}$. By (1), (2), and by $H\left(l_{0}\right)=H\left(m_{0}\right)$ we have

$$
w\left(M, m_{0}^{*}\right) \leq \frac{3}{2} w\left(H\left(m_{0}\right), m_{0}^{*}\right) .
$$

Since the width of a convex body depends continuously on the direction (see $\left[3\right.$, p. 78]) and since $l^{*}$ continuously depends upon $l$, we see that $w\left(M, l^{*}\right)$ is a continuous function of $l$. Thus from (2) and (3), and from the fact that the vertices of $H(l)$ vary continuously as $l$ rotates, we conclude that there is a direction $l_{2}$ such that

$$
w\left(M, l_{2}^{*}\right)=\frac{3}{2} w\left(H\left(l_{2}\right), l_{2}^{*}\right) .
$$

Let $H\left(l_{2}\right)=a_{2} b_{2} c_{2} d_{2} e_{2} f_{2}$. Denote by $l_{1}$ and $l_{3}$ the directions of the vectors $\overrightarrow{b_{2} d_{2}}$ and $\overrightarrow{f_{2} b_{2}}$, respectively. Observe that (1) remains true for $m$ being the direction of $\overrightarrow{f b}$. From both versions of (1) and from (4) we obtain

$$
w\left(M, l_{i}^{*}\right) \leq \frac{3}{2} w\left(H\left(l_{2}\right), l_{i}^{*}\right)
$$

for $i=1$ and $i=3$. This and (4) imply $M \subset \frac{3}{2} H\left(l_{2}\right)$.

The proof is complete.

As observed by a referee, our Lemma is stronger than Theorem 2 of Asplund [1] that $M \subset \frac{3}{2} H$ for an affine-regular hexagon $H \subset M$. We do not see any 


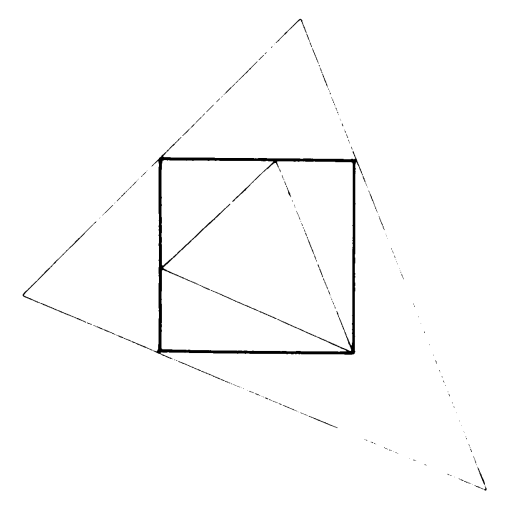

FIGURE 2

way to prove our Theorem directly from Asplund's result; the fact that $H$ is inscribed in $M$ is essential in the proof. By the way, the referee noticed that Asplund proved a stronger result than he stated. His proof indicates that $H$ can be chosen in such a way that $\frac{3}{2} H$ is circumscribed about $M$.

We are ready to confirm one of the conjectures of Grünbaum presented in $[4$, p. 260].

Theorem. Let $M \subset E^{2}$ be a centrally symmetric convex body. We can inscribe in $M$ a triangle $T$ whose centroid is the center of symmetry of $M$ such that $M \subset \frac{5}{2} T$.

Proof. By Lemma it is possible to inscribe in $M$ an affine-regular hexagon $H=a b c d e f$ such that $M \subset \frac{3}{2} H$. Three of the lines containing the sides of $H$ bound a triangle $S_{1}$ containing $H$ and the other three a triangle $S_{2}$. Since $H$ is inscribed in $M$, we see that $M \subset S_{1} \cup S_{2}$.

Of course, the triangle $T$ with the vertices $a, c, e$ is inscribed in $M$.

Denote by $o$ the center of symmetry of $M$. It is easy to see that $o$ is the center of $H$ (see the beginning of the proof of Lemma 1 of [5]). Thus $o$ is the centroid of $T$. Consequently, from the inclusions $M \subset \frac{3}{2} H$ and $M \subset S_{1} \cup S_{2}$ we obtain $M \subset \frac{5}{2} T$, which ends the proof.

The example of $M$ being a parallelogram shows that the ratio $\frac{5}{2}$ in the theorem cannot be lessened (see [4, p. 259]).

In connection with Theorem, the author conjectures that for every convex body $C \subset E^{2}$ there is a triangle $T$ inscribed in $C$ such that $C \subset\left(1+\frac{3}{5} \sqrt{5}\right) T$. The ratio $1+\frac{3}{5} \sqrt{5}$ cannot be improved because of the example of a parallelogram as $C$. (see Figure 2).

We conjecture that for every convex body $C \subset E^{2}$ there is a triangle $T_{1}$ and a homothetic copy $T_{2}$ of $T_{1}$, where the ratio of the homothety is $\cos ^{2} 36^{\circ} / \sin 18^{\circ}$ $=2.118 \ldots$, such that $T_{1} \subset C \subset T_{2}$. An easy but time consuming calculation shows that the above ratio cannot be lessened when $C$ is the regular pentagon (see Figure 3 on next page). Recall that if $C$ is centrally symmetric, the ratio is 2 (see [4, p. 259]). Here is an estimate for an arbitrary convex body $C \subset E^{2}$. 


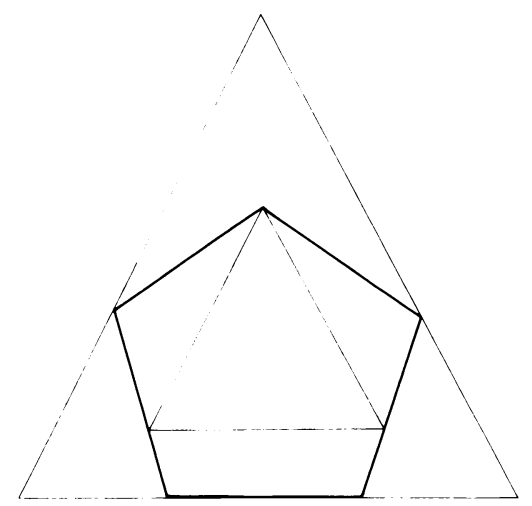

FIGURE 3

Proposition. For every convex body $C \subset E^{2}$ there are a triangle $T_{1}$ and its image $T_{2}$ under a homothety with the ratio $\frac{5}{2}$ such that $T_{1} \subset C \subset T_{2}$.

Proof. Compactness arguments show that we can inscribe in $C$ a triangle $T_{1}$ of maximal area. Let $T_{2}$ be the homothetic copy of $T_{1}$ containing $C$ with the smallest possible positive ratio $\mu$ of the homothety. Of course, there are points $a, b, c$ of $C$ on different sides of $T_{2}$. Let $S=(-2) T_{1}$.

If we suppose that a point $p \in C$ is not in $S$, then a triangle whose vertices are $p$ and two of the vertices of $T_{1}$ is contained in $C$ and has the area greater than the area of $T_{1}$. This contradicts the choice of $T_{1}$ and shows that $C \subset S$.

Of course, $S$ is the copy of $T_{2}$ under a homothety with the negative ratio $-2 / \mu$. Suppose that $\mu>\frac{5}{2}$. Then $-2 / \mu>-\frac{4}{5}$. Thus from $a, b, c \in C \subset$ $S$ we deduce that $a, b, c$ are not contained in the three triangles that are homothetic copies of $T_{2}$ with homothety centers at the vertices of $T_{2}$ and ratio $\frac{1}{5}$. As a consequence, the area of the triangle $a b c$ is greater than $\frac{4}{25}$ of the area of $T_{2}$; i.e., greater than the area of $T_{1}$. This contradiction with the maximality of the area of $T_{1}$ shows that $\mu \leq \frac{5}{2}$.

\section{REFERENCES}

1. E. Asplund, Comparison between plane symmetric convex bodies and parallelograms, Math. Scand. 8 (1960), 171-180.

2. A. S. Besicovitch, Measure of assymetry of convex curves, J. London Math. Soc. 23 (1945), 237-240.

3. H. G. Eggleston, Convexity, Cambridge Univ. Press, Cambridge, 1969.

4. B. Grünbaum, Measures of symmetry of convex sets, Convexity, Proc. Sympos. Pure Math., vol. 7, Amer. Math. Soc., Providence, R. I., 1963, pp. 233-270.

5. M. Lassak, Approximation of convex bodies by centrally symmetric bodies, J. London Math. Soc. (2) 40 (1989), 369-377. 